\title{
Identification of Dutch children diagnosed with atopic diseases using prescription data: a validation study
}

\author{
B Mulder ${ }^{1}$ - F Groenhof ${ }^{2}$ L L I Kocabas ${ }^{1}$ HJ Bos ${ }^{1}$ - TW De Vries ${ }^{3} \cdot$ E Hak ${ }^{1,4}$. \\ CCM Schuiling-Veninga ${ }^{1}$
}

Received: 6 June 2015 / Accepted: 3 September 2015 / Published online: 9 October 2015

(C) The Author(s) 2015. This article is published with open access at Springerlink.com

\begin{abstract}
Purpose The aim of this study is to validate medication proxies for the identification of children diagnosed with atopic disorders that can be applied in various types of epidemiological research.

Methods Records of 7439 children, aged between 0 and 10 years, in the period 2001 until 2010, were retrieved from the Registration Network Groningen database, a general practitioners database in the north-eastern part of the Netherlands. The sensitivity and positive predictive value (PPV) of 22 medication proxies for the identification of children diagnosed with atopic disorders (asthma, atopic dermatitis, and allergic rhinitis) were computed using the registered diagnoses as gold standards. In addition, different capture periods ( 1 year, half year, and length of study period) for the detection of prescriptions were tested for all the medication proxies.

Results The highest PPV $(0.84,95 \%$ CI $0.81-0.87)$ in combination with a sufficient sensitivity value $(0.54,95 \%$ CI $0.50-0.57)$ for the identification of children diagnosed with asthma was yielded for the medication proxy, $\geq 2$ prescriptions
\end{abstract}

B Mulder

b.mulder@rug.nl

1 Department of PharmacoEpidemiology and PharmacoEconomics University Centre of Pharmacy, University of Groningen, P.O. Box XB45, A. Deusinglaan 1, 9713 AV Groningen, The Netherlands

2 Department of General Practice, University of Groningen, University Medical Centre Groningen, Groningen, The Netherlands

3 Department of Pediatrics, Medical Centre Leeuwarden, $\mathrm{H}$ Dunantweg 2, 8934 AD Leeuwarden, The Netherlands

4 Department of Epidemiology, University Medical Centre Groningen, Hanzeplein 1, P.O. Box 30.001, 9700

RB Groningen, The Netherlands for anti-asthma medication within 1 year, including 1 inhaled steroid. PPV and sensitivity were even higher in the age group 6-10 years. The proxies designed for the identification of children diagnosed with atopic dermatitis and allergic rhinitis yielded only high PPVs $(\geq 0.75)$ in combination with low sensitivity values $(\leq 0.22)$. Altering the capture period for the detection of prescriptions to half a year or the length of the study period only affected sensitivity values.

Conclusion Children diagnosed with asthma can be identified reliably with a range of medication proxies. The use of prescription data for the identification of children diagnosed with atopic dermatitis and allergic rhinitis is questionable.

Keywords Prescriptions $\cdot$ Specificity $\cdot$ Sensitivity $\cdot$ Atopic diseases $\cdot$ Asthma $\cdot$ Eczema $\cdot$ Hay fever

\section{Introduction}

Pharmacy databases with large numbers of prescriptions can provide valuable information for observational studies [1]. However, the validity of using prescription data for the identification of children with atopic diseases in epidemiological research has been questioned, especially in children [2-8].

Various efforts have already been made to validate methods for the identification of asthma patients with prescription data [3-8]. A former study of our group investigated the accuracy of the use of several medication proxies for the identification of asthma patients (age 19-49) and showed that asthma patients could be identified reliably from prescription data [5]. However, results from an adult patient population cannot directly be generalized to a population of children, especially not in the case of asthma [9]. Making an asthma 
diagnosis in children is difficult since objective lung tests cannot be performed in patients under the age of 6 [9]. In addition, children may use anti-asthma medication for other indications than adults (acute bronchitis versus chronic obstructive pulmonary disease) [2]. Recent studies developed a valid method for the identification of asthmatic children (age 4.5-17 years) using prescription data $[3,7,8]$. However, for different study purposes, different accuracy measures (sensitivity, specificity, positive predictive value, negative predictive value) are important and hence various proxies are needed to be developed for the use in various epidemiological studies [10]. In addition, children with asthma often have concomitant allergic diseases like atopic dermatitis or allergic rhinitis, due to common pathogenesis [11]. To our knowledge, only one study has focused on the validation of an identification tool for children with atopic dermatitis and concluded that the identification of children with this disease based on a medication proxy was insufficient [8]. Since only one medication proxy was tested and only positive predictive values were reported, there is still need for the validation of other proxies for atopic dermatitis. In addition, no studies have focused on the identification of children diagnosed with allergic rhinitis.

Therefore, the main objective of this study was to determine reliable medication proxies for the identification of children diagnosed with atopic diseases (asthma, atopic dermatitis, and rhinitis) applicable in different types of epidemiological research.

\section{Methods}

\section{Setting}

This register-based cross-sectional study was performed with data from the Registration Network Groningen (RNG) database. This network was established in 1989 and consists of patient registrations of three general practices with 17 general practitioners (GPs) in the north-eastern part of the Netherlands. The RNG includes a dynamic population with an average annual population of approximately 30,000 patients. Patients were anonymized and identified with a unique patient number. This network contains information about patient characteristics, diagnoses, and prescription records for each patient. Each prescription contains an anatomical therapeutic chemical code (ATC-code), and each diagnosis is described with the International Classification for Primary Care (ICPC) code [12]. GPs are trained specifically to work with the coding system, and coding of ICPC and ATC codes was proven to be accurate in previous studies [7, 13]. In the Netherlands, patients are registered to a single GP, so records in the RNG database can be assumed to be complete for the individual patient [14]. Diagnoses and prescriptions from specialists are included in the database if these are communicated to one of the GPs by a so-called retour letter. More information about the database is described elsewhere $[15,16]$.

\section{Study population}

Patient records from 01 January 2001 until 31 December 2010 were selected from the RNG database. Patients were included in the study if they had at least one physician encounter (visit, telephone consultation, or prescription request) during the study period and were aged between 0 and 10 years at the moment of encounter.

\section{Prescription data}

Data were obtained for all prescriptions regarding drugs for obstructive airway diseases (ATC R03), dermatological preparations with corticosteroids (ATC D07), other agents for the treatment of dermatitis (ATC D11AH), nasal preparations (R01), and systemic antihistamines (ATC R06) prescribed from 01 January 2001 until 31 December 2010. Twenty-two medication proxies (listed in Table 1) with a capture period of 1 year were designed for the identification of children diagnosed with atopic diseases, according to the Dutch guidelines for general practitioners [17-19]. The accuracy measures sensitivity and PPV were calculated for all medication proxies. Specificity and NPV were not calculated since these values will be artificially high, due to the large number of non-allergic patients included in the study population [5].

\section{Analysis}

A recorded diagnosis of either atopic dermatitis (ICPC S87), asthma (ICPC R96), or allergic rhinitis (ICPC R97) during the study period was defined as the gold standard. For each medication proxy, the sensitivity, positive predictive value (PPV), and $95 \%$ confidence intervals $(95 \% \mathrm{CI})$ were calculated. In additional analyses, different capture periods for the detection of prescriptions (half year and total study period) were tested for all the medication proxies. Since the PPV is dependent on the prevalence of the indication in a population, a sensitivity analysis was performed for the proxy $\geq 2$ inhaled steroids within a year. In this extra analysis, positive predictive values were calculated for a reasonable range of prevalence numbers of asthma, derived from an ISAAC study into the worldwide trends in asthma prevalence [20]. All analyses were conducted using the IBM SPSS Statistics 20 version. 
Table 1 Medication proxies used for the identification of children diagnosed with the atopic disorders asthma, atopic dermatitis, and allergic rhinitis

\begin{tabular}{|c|c|c|}
\hline Indication (ICPC code) & $\mathrm{nr}$ & Medication proxies \\
\hline \multirow[t]{8}{*}{ Asthma (ICPC R96) } & 1 & $\geq 1$ prescription for an inhaled anti-asthma drug (ATC R03) \\
\hline & 2 & $\geq 1$ prescription for an inhaled corticosteroid (ATC R03AK, R03BA) \\
\hline & 3 & $\geq 2$ prescriptions for an inhaled anti-asthma drug (ATC R03) \\
\hline & 4 & $\geq 1$ inhaled steroid (ATC R03AK, R03BA) $+\geq 1$ inhaled other anti-asthma drug (ATC R03) \\
\hline & 5 & $\geq 3$ prescriptions for an inhaled anti-asthma drug (ATC R03) \\
\hline & 6 & $\geq 2$ prescriptions for an inhaled corticosteroid (ATC R03AK, R03BA) \\
\hline & 7 & $\geq 4$ prescriptions for an inhaled anti-asthma drug (ATC R03) \\
\hline & 8 & $\geq 5$ prescriptions for an inhaled anti-asthma drug (ATC R03) \\
\hline \multirow[t]{6}{*}{ Atopic dermatitis (ICPC S87) } & 9 & $\geq 1$ prescription for a dermal steroid (ATC D07) \\
\hline & 10 & $\geq 1$ prescription for an ointment with an immunosuppressant (ATC D11) \\
\hline & 11 & $\geq 2$ prescriptions for a dermal steroid (ATC D07) \\
\hline & 12 & $\geq 3$ prescriptions for a dermal steroid (ATC D07) \\
\hline & 13 & $\geq 4$ prescriptions for a dermal steroid (ATC D07) \\
\hline & 14 & $\geq 5$ prescriptions for a dermal steroid (ATC D07) \\
\hline \multirow[t]{8}{*}{ Allergic rhinitis (ICPC R97) } & 15 & $\geq 1$ prescription for a systemic antihistamine (ATC R06) \\
\hline & 16 & $\geq 1$ prescription for a nasal antihistamine (ATC R01AC) \\
\hline & 17 & $\geq 1$ prescription for a nasal corticosteroid (ATC R01AD) \\
\hline & 18 & $\begin{array}{l}\geq 2 \text { prescriptions for systemic antihistamines (ATC R06), nasal antihistamines (ATC R01AC) } \\
\text { or nasal steroids (ATC R01AD) }\end{array}$ \\
\hline & 19 & $\begin{array}{l}\geq 3 \text { prescriptions for systemic antihistamines (ATC R06), nasal antihistamines (ATC R01AC) } \\
\text { or nasal steroids (ATC R01AD) }\end{array}$ \\
\hline & 20 & $\geq 2$ prescriptions for a nasal corticosteroid (ATC R01AD) \\
\hline & 21 & $\begin{array}{l}\geq 4 \text { prescriptions for systemic antihistamines (ATC R06), nasal antihistamines (ATC R01AC) } \\
\text { or nasal steroids (ATC R01AD) }\end{array}$ \\
\hline & 22 & $\begin{array}{l}\geq 5 \text { prescriptions for systemic antihistamines (ATC R06), nasal antihistamines (ATC R01AC) } \\
\text { or nasal steroids (ATC R01AD) }\end{array}$ \\
\hline
\end{tabular}

\section{Results}

In total, 7439 children until the age of 10 years were included in the study. Of these children, $1835(24.7 \%)$ had a registered diagnosis of an allergic disorder (asthma, atopic dermatitis, or allergic rhinitis) in their records. The diagnosis asthma (ICPC R96) was registered in 809 (10.9\%) children (Table 2), atopic dermatitis (ICPC S87) in 985 (13.2\%) children (Table 3), and allergic rhinitis (ICPC R97) in 419 (5.6\%) children (Table 4). Drugs for obstructive airway disease (ATC R03) were used by 744 (10.0\%) children, dermal corticosteroids (ATC D07) by 1990 (26.8 \%) children, and nasal antihistamines (ATC R01AC) or nasal corticosteroids (R01AD) by 518 (7.0\%) children.

\section{Validation of the medication proxies for the identification of children diagnosed with asthma}

In Table 5, the sensitivity and PPV of the eight medication proxies for the identification of children diagnosed with asthma are shown. When a capture period of 1 year was applied, medication proxy 1 , receiving $\geq 1$ prescription for any anti- asthmatic drug, yielded the highest sensitivity of $0.92(95 \%$ CI 0.90-0.94). However, only half of the identified children with this proxy had a registered diagnosis of asthma (PPV $0.54,95 \%$ CI $0.51-0.57)$. Table 2 shows that of the children that got prescribed anti-asthma drugs, $28.2 \%$ had a diagnosis of acute bronchitis and/or $24.3 \%$ a diagnosis of cough without a concomitant asthma diagnosis. Of the children who got prescribed inhaled corticosteroids, only 16.8 and $14.3 \%$ had a diagnosis of acute bronchitis or cough without a concomitant asthma diagnosis, respectively (Table 2). Proxies that included inhaled corticosteroids ( $\mathrm{nr} 2,4$, and 6 ) yielded therefore higher PPVs $(0.76,0.84$, and 0.87 , respectively) for the identification of children with an asthma diagnosis. In addition, including $\geq 2$ prescriptions for anti-asthma drugs increased the PPV as well (Fig. 1).

\section{Validation of the medication proxies for the identification of children diagnosed with atopic dermatitis}

In Table 5, the sensitivity and PPV of the six medication proxies for the identification of children diagnosed with atopic dermatitis are shown. When a capture period of 1 year was 
applied, medication proxy 9 , receiving at least 1 prescription for dermal corticosteroids, yielded the highest sensitivity of 0.80 (95\% CI $0.77-0.82$ ). However, only $39 \%$ of the identified children had a registered diagnosis of atopic dermatitis (PPV 0.39, $95 \%$ CI 0.37-0.41). Table 3 shows that of the children who got prescribed dermal corticosteroids, $37.6 \%$ had been diagnosed with the ICPC code for contact dermatitis/other dermatitis. Medication proxy 13, receiving at least 4 prescriptions for dermal steroids within a year, yielded a higher PPV of 0.75 (95\% CI 0.65-0.85). However, only $12 \%$ of the children diagnosed with atopic dermatitis were detected.

\section{Validation of the medication proxies for the identification of children diagnosed with allergic rhinitis}

In Table 5, the sensitivity and PPV of the nine medication proxies for the identification of children diagnosed with allergic rhinitis are shown. When a capture period of 1 year was applied, medication proxy 15 , receiving at least 1 prescription for a anithistamine, yielded the highest sensitivity of 0.72 (95\% CI 0.68-0.76). However, only $23 \%$ of the identified children had a diagnosis of allergic rhinitis (PPV 0.23, $95 \%$ CI $0.20-0.25$ ). Medication proxy 16 , receiving at least 1 prescription for nasal antihistamine, yielded a higher PPV of 0.87 (95\% CI $0.81-0.93$ ). However, only $22 \%$ of the children diagnosed with allergic rhinitis were detected. Table 4 showed that of the children who got prescribed nasal antihistamines, $27.2 \%$ received the diagnosis asthma without a concomitant diagnosis of allergic rhinitis.

Variation of the capture period for the detection of prescriptions had more effect on sensitivity values (maximum change $400 \%$ for proxy 14) than that on the PPVs (maximum change of $18 \%$ for proxy 20 ) for the identification of children diagnosed with asthma, atopic dermatitis, and allergic rhinitis.

Additional analyses, in which the population was stratified by age, showed that accuracy measures of the proxies designed for the identification of children diagnosed with atopic dermatitis were higher in the age group $0-5$ years (Table 6). On the contrary, accuracy measures of the proxies designed for the identification of children diagnosed with asthma and allergic rhinitis were higher in the age group 6-10 years (Table 6).

\section{Sensitivity analyses}

The results of the sensitivity analyses on the PPVs of the proxies $\geq 2$ inhaled steroids in a year were presented in Fig. 2. This figure showed that the PPV for the identification of children diagnosed with asthma was accurate for a reasonable range of asthma prevalence numbers [20]. 


\section{Discussion}

The current study showed that accuracy measures of the medication proxies for asthma were relatively high, especially in children older than 5 years. This suggests that children in this age group diagnosed with asthma can be identified reliably with prescription data. On the contrary, sensitivity and positive predictive values of the proxies designed for the identification of children diagnosed with atopic dermatitis and rhinitis were lower. The use of prescription data for the identification of children diagnosed with atopic dermatitis and allergic rhinitis is therefore questionable. This study provides different medication proxies to aid various epidemiological studies with the identification of children diagnosed with atopic diseases.

\section{Interpretation and comparison with literature}

Of the different allergic diseases, medication proxies designed for the identification of asthmatic children yielded highest accuracy measures, especially for children who were diagnosed beyond the age of 5 . The evaluation of asthma in young children is complicated by the lack of objective lung function measurements [9], explaining the slightly better prediction of an asthma diagnosis in the older age group. In addition, antiasthmatic medication was prescribed for other respiratory conditions, like acute bronchitis and cough. Results showed that a better distinction between different respiratory conditions can be made with the inclusion of inhaled corticosteroids in the proxy. In addition, medication proxies can yield higher PPVs if the proxy includes multiple prescriptions for inhaled asthma drugs. Accuracy measures of our medication proxies for the identification of children diagnosed with asthma were comparable with other validation studies $[3,7,8]$. However, our study showed better accuracy measures in the age group $0-5$ than any of the previously performed studies when the proxy with $\geq 2$ prescriptions for inhaled steroids was applied. Though asthma cannot be diagnosed objectively before the age of 5 , in the Netherlands, it is a common practice to diagnose children with recurrent wheeze as being asthmatic. This may explain the slightly better accuracy measures in the current study. In addition, the majority of the medication proxies presented in the current study included the total group of anti-asthma medication rather than specific subtypes of anti-asthma medication. According to previous studies, the PPV may even be higher if medication proxies included more specific subgroups of anti-asthma drugs [3]. The medication proxies for the detection of children with atopic dermatitis yielded lower accuracy measures. This can mainly be explained by the use of dermal steroids for other indications than atopic dermatitis, like the indication contact dermatitis/other dermatitis (ICPC S88). Since atopic dermatitis is often treated for a longer duration than other types of dermatitis, differentiation between 
Table 4 Number of patients aged 0-10 years that received medication for the treatment of allergic rhinitis, stratified by diagnosis

\begin{tabular}{|c|c|c|c|c|}
\hline \multirow[b]{3}{*}{ Type of medication } & & \multicolumn{3}{|l|}{ Diagnosis } \\
\hline & & \multirow{2}{*}{$\begin{array}{l}\text { Allergic rhinitis } \\
\text { Allergic rhinitis ICPC } \\
\text { R97 }(N=419)\end{array}$} & \multicolumn{2}{|c|}{ Other diseases without concomitant allergic rhinitis } \\
\hline & & & $\begin{array}{l}\text { Asthma ICPC R97 } \\
(N=690)\end{array}$ & $\begin{array}{l}\text { Sneezing/cold ICPC } \\
\text { R07 }(N=263)\end{array}$ \\
\hline Nasal preparations (R01) & 752 & $238(31.6)$ & $99(13.2)$ & $85(11.3)$ \\
\hline Nasal anti-allergic preparations (R01AC) & 162 & $103(63.6)$ & $44(27.2)$ & $6(3.7)$ \\
\hline Nasal steroids (R01AD) & 356 & $152(42.7)$ & $43(12.1)$ & $51(14.3)$ \\
\hline Systemic antihistamines (R06) & 1332 & $301(22.6)$ & $195(14.6)$ & $58(4.4)$ \\
\hline
\end{tabular}

Numbers and percentages do not sum up to $100 \%$, due to multiple medications and diagnoses

ICPC International Classification for Primary Care

indications can only be made with a medication proxy that resembles a long-term treatment $(\geq 4$ prescriptions for dermal steroids). The medication proxy of 4 prescriptions for dermal corticosteroids within the total time of the study period yielded an acceptable PPV of 0.75 in the age group 0-10 and an even higher PPV in the age group 0-5. However, the sensitivity of

Table 5 Accuracy measures of medication proxies for the identification of children ( $0-10$ years) diagnosed with asthma, atopic dermatitis, and allergic rhinitis

\begin{tabular}{|c|c|c|c|c|c|c|c|}
\hline & & \multicolumn{2}{|c|}{ Half-year capture period } & \multicolumn{2}{|c|}{ 1-year capture period } & \multicolumn{2}{|c|}{ Within study period } \\
\hline \multicolumn{2}{|c|}{ Medication proxies } & (95\% CI) & $(95 \% \mathrm{CI})$ & (95\% CI) & $(95 \% \mathrm{CI})$ & (95 \% CI) & $(95 \% \mathrm{CI})$ \\
\hline \multicolumn{8}{|c|}{ Asthma } \\
\hline 1 & $\geq 1$ inhaled anti-asthma drugs & $0.92(0.90-0.94)$ & $0.54(0.51-0.57)$ & $0.92(0.90-0.94)$ & $0.54(0.51-0.57)$ & $0.92(0.90-0.94)$ & $0.54(0.51-0.57)$ \\
\hline 2 & $\geq 1$ inhaled steroid & $0.62(0.59-0.66)$ & $0.76(0.73-0.80)$ & $0.62(0.59-0.66)$ & $0.76(0.73-0.80)$ & $0.62(0.59-0.66)$ & $0.76(0.73-0.80)$ \\
\hline 3 & $\geq 2$ inhaled anti-asthma drugs & $0.65(0.62-0.69)$ & $0.75(0.72-0.79)$ & $0.69(0.66-0.73)$ & $0.74(0.70-0.77)$ & $0.75(0.72-0.78)$ & $0.71(0.68-0.74)$ \\
\hline 4 & $\begin{array}{l}\geq 1 \text { inhaled steroid }+\geq 1 \text { inhaled } \\
\text { other anti-asthma drug }\end{array}$ & $0.52(0.49-0.55)$ & $0.85(0.82-0.88)$ & $0.54(0.50-0.57)$ & $0.84(0.81-0.87)$ & $0.55(0.51-0.58)$ & $0.83(0.80-0.87)$ \\
\hline 5 & $\geq 3$ inhaled anti-asthma drugs & $0.45(0.42-0.49)$ & $0.83(0.80-0.87)$ & $0.54(0.50-0.57)$ & $0.82(0.79-0.85)$ & $0.63(0.60-0.67)$ & $0.80(0.77-0.83)$ \\
\hline 6 & $\geq 2$ inhaled steroids & $0.43(0.39-0.46)$ & $0.87(0.84-0.91)$ & $0.46(0.43-0.49)$ & $0.87(0.83-0.90)$ & $0.49(0.46-0.53)$ & $0.85(0.82-0.89)$ \\
\hline 7 & $\geq 4$ inhaled anti-asthma drugs & $0.33(0.30-0.36)$ & $0.88(0.84-0.92)$ & $0.44(0.40-0.47)$ & $0.86(0.83-0.90)$ & $0.53(0.50-0.57)$ & $0.83(0.80-0.87)$ \\
\hline 8 & $\geq 5$ inhaled anti-asthma drugs & $0.23(0.20-0.26)$ & $0.90(0.86-0.94)$ & $0.35(0.32-0.38)$ & $0.90(0.87-0.93)$ & $0.48(0.45-0.51)$ & $0.87(0.83-0.90)$ \\
\hline \multicolumn{8}{|c|}{ Atopic dermatitis } \\
\hline 9 & $\geq 1$ dermal steroid & $0.80(0.77-0.82)$ & $0.40(0.38-0.42)$ & $0.80(0.77-0.82)$ & $0.40(0.38-0.42)$ & $0.80(0.77-0.82)$ & $0.40(0.38-0.42)$ \\
\hline 10 & $\geq 1$ immunosuppressant & $0.01(0.01-0.02)$ & $0.71(0.49-0.92)$ & $0.01(0.01-0.02)$ & $0.71(0.49-0.92)$ & $0.01(0.01-0.02)$ & $0.71(0.49-0.92)$ \\
\hline 11 & $\geq 2$ dermal steroids & $0.33(0.30-0.36)$ & $0.60(0.56-0.64)$ & $0.40(0.37-0.43)$ & $0.60(0.56-0.64)$ & $0.50(0.47-0.53)$ & $0.57(0.54-0.60)$ \\
\hline 12 & $\geq 3$ dermal steroids & $0.14(0.12-0.16)$ & $0.71(0.64-0.77)$ & $0.19(0.16-0.21)$ & $0.67(0.61-0.72)$ & $0.31(0.28-0.34)$ & $0.64(0.60-0.69)$ \\
\hline 13 & $\geq 4$ dermal steroids & $0.06(0.04-0.07)$ & $0.74(0.64-0.84)$ & $0.12(0.10-0.14)$ & $0.75(0.65-0.85)$ & $0.20(0.18-0.23)$ & $0.71(0.65-0.76)$ \\
\hline 14 & $\geq 5$ dermal steroids & $0.03(0.02-0.04)$ & $0.74(0.61-0.88)$ & $0.06(0.04-0.07)$ & $0.78(0.71-0.85)$ & $0.15(0.13-0.17)$ & $0.77(0.71-0.83)$ \\
\hline \multicolumn{8}{|c|}{ Rhinitis } \\
\hline 15 & $\geq 1$ systemic antihistamine & $0.72(0.68-0.76)$ & $0.23(0.20-0.25)$ & $0.72(0.68-0.76)$ & $0.23(0.20-0.25)$ & $0.72(0.68-0.76)$ & $0.23(0.20-0.25)$ \\
\hline 16 & $\geq 1$ nasal antihistamine & $0.22(0.18-0.26)$ & $0.87(0.81-0.93)$ & $0.22(0.18-0.26)$ & $0.87(0.81-0.93)$ & $0.22(0.18-0.26)$ & $0.87(0.81-0.93)$ \\
\hline 17 & $\geq 1$ nasal steroid & $0.36(0.32-0.41)$ & $0.43(0.38-0.48)$ & $0.36(0.32-0.41)$ & $0.43(0.38-0.48)$ & $0.36(0.32-0.41)$ & $0.43(0.38-0.48)$ \\
\hline 18 & $\begin{array}{r}\geq 2 \text { systemic antihistamines, nasal } \\
\text { antihistamines or nasal steroids }\end{array}$ & $0.53(0.49-0.58)$ & $0.47(0.42-0.51)$ & $0.59(0.54-0.64)$ & $0.43(0.39-0.47)$ & $0.67(0.62-0.71)$ & $0.40(0.36-0.43)$ \\
\hline 19 & $\begin{array}{r}\geq 3 \text { systemic antihistamines, nasal } \\
\text { antihistamines or nasal steroids }\end{array}$ & $0.34(0.29-0.38)$ & $0.61(0.54-0.67)$ & $0.40(0.35-0.45)$ & $0.57(0.51-0.62)$ & $0.52(0.47-0.57)$ & $0.50(0.46-0.55)$ \\
\hline 20 & $\geq 2$ nasal steroids & $0.16(0.12-0.19)$ & $0.59(0.50-0.68)$ & $0.18(0.14-0.22)$ & $0.60(0.51-0.68)$ & $0.20(0.16-0.24)$ & $0.57(0.49-0.65)$ \\
\hline 21 & $\begin{array}{l}\geq 4 \text { systemic antihistamines, nasal } \\
\text { antihistamines or nasal steroids }\end{array}$ & $0.19(0.15-0.23)$ & $0.67(0.58-0.75)$ & $0.27(0.23-0.31)$ & $0.63(0.56-0.70)$ & $0.40(0.36-0.45)$ & $0.58(0.52-0.64)$ \\
\hline 22 & $\begin{array}{l}\geq 5 \text { systemic antihistamines, nasal } \\
\text { antihistamines or nasal steroids }\end{array}$ & $0.13(0.10-0.16)$ & $0.71(0.60-0.81)$ & $0.18(0.15-0.22)$ & $0.65(0.56-0.73)$ & $0.34(0.30-0.39)$ & $0.62(0.56-0.69)$ \\
\hline
\end{tabular}


Fig. 1 Development of sensitivity and positive predictive value (PPV) with an increasing number of anti-asthma prescriptions within 1 year

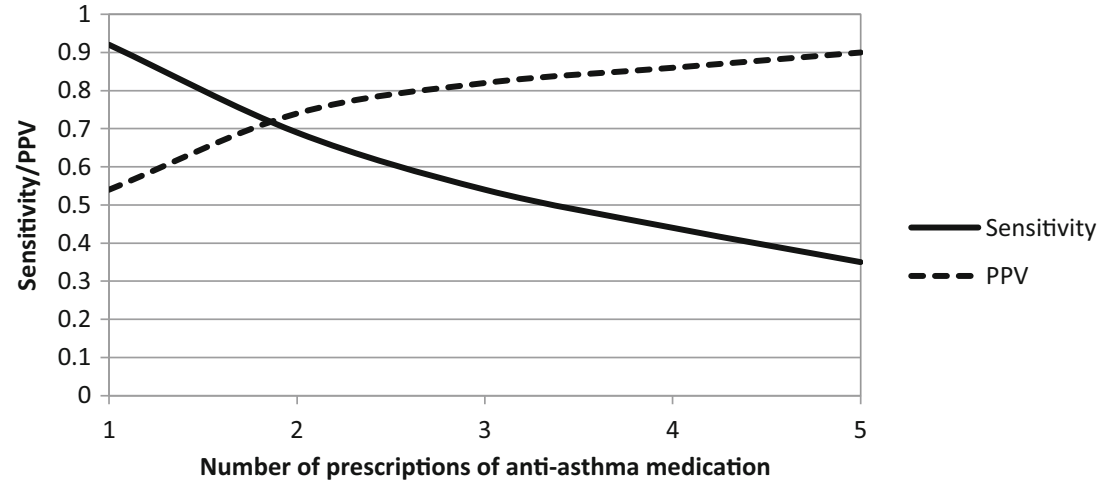

this proxy was really low $(0.12)$. Hence, consideration should be made if its use is feasible, since only a really small percentage of children with a diagnosis of atopic dermatitis will be detected. This medication proxy may only be applied in large databases. Only one previous study validated a medication proxy for the identification of children diagnosed with atopic dermatitis and reported a PPV of 0.45 [8]. The PPV of the current study is much higher (0.75). This can mainly be

Table 6 Accuracy measures of medication proxies for the identification of children diagnosed with asthma, atopic dermatitis, and allergic rhinitis for children aged $0-5$ and children aged 5-10

\begin{tabular}{|c|c|c|c|c|c|}
\hline & \multirow[b]{3}{*}{ Medication proxies } & \multicolumn{4}{|c|}{ 1-year capture period } \\
\hline & & \multicolumn{2}{|l|}{$0-5$ years } & \multicolumn{2}{|l|}{$6-10$ years } \\
\hline & & $\begin{array}{l}\text { Sensitivity } \\
(95 \% \mathrm{CI})\end{array}$ & $\begin{array}{l}\text { PPV } \\
(95 \% \text { CI })\end{array}$ & $\begin{array}{l}\text { Sensitivity } \\
(95 \% \mathrm{CI})\end{array}$ & $\begin{array}{l}\text { PPV } \\
(95 \% \text { CI })\end{array}$ \\
\hline & \multicolumn{5}{|l|}{ Asthma } \\
\hline 1 & $\geq 1$ inhaled anti-asthma drugs & $0.90(0.87-0.92)$ & $0.48(0.45-0.51)$ & $0.94(0.91-0.96)$ & $0.71(0.67-0.75)$ \\
\hline 2 & $\geq 1$ inhaled steroid & $0.58(0.54-0.62)$ & $0.71(0.67-0.75)$ & $0.68(0.63-0.72)$ & $0.84(0.80-0.88)$ \\
\hline 3 & $\geq 2$ inhaled anti-asthma drugs & $0.64(0.60-0.68)$ & $0.67(0.63-0.71)$ & $0.75(0.71-0.79)$ & $0.85(0.81-0.89)$ \\
\hline 4 & $\geq 1$ inhaled steroid $+\geq 1$ inhaled other anti-asthma drug & $0.49(0.45-0.53)$ & $0.79(0.75-0.84)$ & $0.57(0.52-0.61)$ & $0.89(0.85-0.93)$ \\
\hline 5 & $\geq 3$ inhaled anti-asthma drugs & $0.49(0.45-0.53)$ & $0.77(0.73-0.81)$ & $0.58(0.53-0.63)$ & $0.88(0.84-0.92)$ \\
\hline 6 & $\geq 2$ inhaled steroids & $0.43(0.39-0.47)$ & $0.81(0.77-0.86)$ & $0.51(0.46-0.56)$ & $0.92(0.89-0.96)$ \\
\hline 7 & $\geq 4$ inhaled anti-asthma drugs & $0.39(0.35-0.43)$ & $0.82(0.77-0.87)$ & $0.50(0.45-0.54)$ & $0.91(0.87-0.95)$ \\
\hline 8 & $\geq 5$ inhaled anti-asthma drugs & $0.30(0.26-0.34)$ & $0.85(0.81-0.90)$ & $0.40(0.35-0.45)$ & $0.94(0.90-0.97)$ \\
\hline \multicolumn{6}{|c|}{ Atopic dermatitis } \\
\hline 9 & $\geq 1$ dermal steroid & $0.78(0.75-0.81)$ & $0.45(0.43-0.48)$ & $0.77(0.72-0.82)$ & $0.29(0.26-0.33)$ \\
\hline 10 & $\geq 1$ immunosuppressant & $0.01(0.00-0.02)$ & $0.78(0.51-1.05)$ & $0.02(0.00-0.03)$ & $0.63(0.29-0.96)$ \\
\hline 11 & $\geq 2$ dermal steroids & $0.40(0.36-0.43)$ & $0.65(0.61-0.70)$ & $0.37(0.31-0.42)$ & $0.48(0.41-0.54)$ \\
\hline 12 & $\geq 3$ dermal steroids & $0.19(0.16-0.22)$ & $0.72(0.66-0.78)$ & $0.15(0.11-0.19)$ & $0.52(0.41-0.62)$ \\
\hline 13 & $\geq 4$ dermal steroids & $0.12(0.10-0.14)$ & $0.71(0.60-0.82)$ & $0.09(0.06-0.12)$ & $0.71(0.57-0.85)$ \\
\hline 14 & $\geq 5$ dermal steroids & $0.06(0.04-0.07)$ & $0.71(0.60-0.82)$ & $0.04(0.02-0.06)$ & $0.72(0.52-0.93)$ \\
\hline \multicolumn{6}{|c|}{ Rhinitis } \\
\hline 15 & $\geq 1$ systemic antihistamine & $0.71(0.63-0.78)$ & $0.12(0.09-0.14)$ & $0.70(0.65-0.75)$ & $0.33(0.30-0.37)$ \\
\hline 16 & $\geq 1$ nasal antihistamine & $0.11(0.05-0.16)$ & $0.78(0.59-0.97)$ & $0.25(0.21-0.30)$ & $0.90(0.83-0.96)$ \\
\hline 17 & $\geq 1$ nasal steroid & $0.20(0.13-0.26)$ & $0.28(0.19-0.37)$ & $0.38(0.33-0.44)$ & $0.45(0.39-0.51)$ \\
\hline 18 & $\geq 2$ systemic antihistamines, nasal antihistamines or nasal steroids & $0.47(0.38-0.55)$ & $0.23(0.18-0.28)$ & $0.61(0.56-0.67)$ & $0.55(0.50-0.60)$ \\
\hline 19 & $\geq 3$ systemic antihistamines, nasal antihistamines or nasal steroids & $0.30(0.22-0.38)$ & $0.35(0.26-0.44)$ & $0.42(0.37-0.47)$ & $0.64(0.58-0.71)$ \\
\hline 20 & $\geq 2$ nasal steroids & $0.11(0.05-0.16)$ & $0.61(0.41-0.81)$ & $0.19(0.15-0.23)$ & $0.57(0.47-0.66)$ \\
\hline 21 & $\geq 4$ systemic antihistamines, nasal antihistamines or nasal steroids & $0.16(0.10-0.22)$ & $0.36(0.24-0.49)$ & $0.33(0.25-0.35)$ & $0.68(0.61-0.76)$ \\
\hline 22 & $\geq 5$ systemic antihistamines, nasal antihistamines or nasal steroids & $0.11(0.06-0.17)$ & $0.42(0.26-0.58)$ & $0.21(0.17-0.25)$ & $0.70(0.61-0.79)$ \\
\hline
\end{tabular}


Fig. 2 Sensitivity of the PPV for childhood asthma as a function of the prevalence for a range of values [20]. Positive predictive values were derived from the proxy $\geq 2$ inhaled steroids in a year

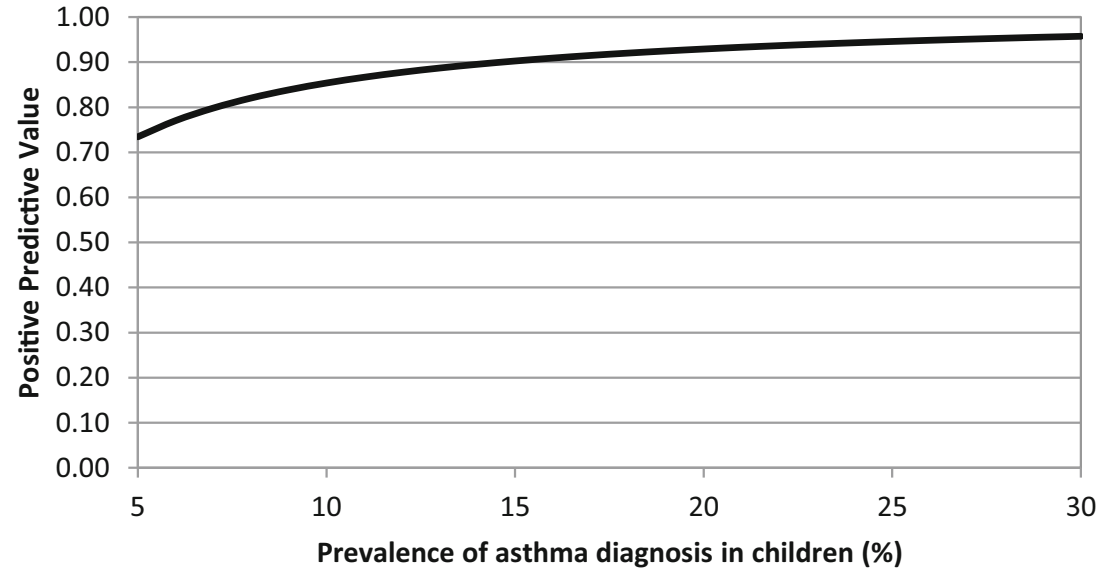

explained by the fact that the medication proxy designed in the current study resembled long-term treatment.

This is the first study that validated medication proxies designed for the identification of children diagnosed with allergic rhinitis. The medication proxy $\geq 1$ prescription for nasal antihistamines yielded a high PPV of 0.87 (95\% CI 0.81$0.93)$. However, similar to the medication proxy for atopic dermatitis, the sensitivity is low (0.22) and its use is questionable.

\section{Strengths and limitations}

The major strength of the current study is the validity testing of a wide range of medication proxies for the identification of children diagnosed with different atopic diseases. Previous studies that validated medication proxies for the identification of children with asthma mainly validated proxies applicable in etiological research and focused on PPVs. Though many studies focus on etiological research, proxies which prioritize sensitivity over PPV may also be useful. To our knowledge, this is the first study that validated medication proxies for the identification of children diagnosed with allergic rhinitis and the second to validate the identification of children diagnosed with atopic dermatitis with prescribing data.

The RNG database is representative for the Netherlands as a whole, and results are generalizable to the Dutch childhood population. Since prescribing guidelines differ between countries, it should be noted that findings from our study may be at least in part specific for the situation in the Netherlands. Though misclassification can occur in every database, the coding of ICPC and ATC codes by the GPs in the RNG database was proven to be accurate by previous studies [7, 13]. Though GPs in the RNG database have been trained specifically to work with the coding system, behavior of diagnosing and prescribing may vary between practices and influence accuracy measures of the medication proxies. Nonetheless, additional analyses detected no difference between the practices (percentages of truly positive identified asthma patients varied from 8.6 until $11.1 \%$ between practices) in identifying diagnosed asthmatic children with the medication proxy $\geq 1$ prescription for anti-asthma drugs (data on file).

\section{Implications for future research}

The selection of a particular medication proxy will always depend on the focus of interest of a study and the available data sources. Though studies that require maximizing one accuracy measure completely at the expense of another are rare, there are situations in which one accuracy measure may be more important than the other [10]. In the following scenarios, we discuss the applicability of the presented proxies for future research.

The sensitivity of a proxy may be given more importance if the goal is to identify all patients with a certain condition in a population. The proxy $\geq 1$ prescription for anti-asthmatic drugs detects $92 \%$ of the children with an asthma diagnosis. As a consequence, $46 \%$ of the included cases are false positives. This may not be a problem if additional verification, like a personal interview, takes place after inclusion. Another scenario that requires a proxy with a high sensitivity is if the aim is to assess the full range of disease outcomes rather than only the most severe. Less sensitive proxies may detect only the severe outcomes, so results may not be generalizable to the whole patient population. This may be important when, for example, a study focusses on the effectiveness of asthma treatment. A proxy with a low sensitivity may fail to detect the effectiveness of asthma treatment that works for mild but not for severe asthma cases. For atopic dermatitis and allergic rhinitis, medication proxies that yielded acceptable sensitivity values yielded low PPVs. The applicability of these proxies for future research is questionable, since more than half of the included patients will be false positives. Therefore, additional verification of the condition should take place after inclusion.

The PPV of a proxy may be given more importance over the sensitivity of a proxy when a study aims to identify only patients that truly have the condition, rather than be 
representative of all persons that have the condition. In etiological studies, researchers want to ensure that the children that are defined as cases do actually have the disease [21]. A proxy with a low PPV includes more false positives and may fail to detect an association between the exposure and the outcome, since this would bias the effect estimate towards the null. For asthma, the proxy $\geq 2$ inhaled steroids within a year can be applied since it yielded a high PPV (0.87) and still half of the patients diagnosed with asthma were detected. However, the proxies that yielded satisfactory PPVs for atopic dermatitis and allergic rhinitis yielded low sensitivity values. Hence, it should be considered if its use is feasible, since only a really small percentage of children with a diagnosis of these conditions will be detected. Since the PPV is an indirect accuracy measure and dependent on the sensitivity and specificity, it cannot be influenced directly by the researcher. In addition, the PPV is influenced by the prevalence of the condition, which may vary between different populations. However, sensitivity analyses of varying prevalence of the disease on the PPV of the proxy showed that PPVs were satisfactory over a reasonable range of prevalence numbers of asthma (Fig. 2).

Since the specificity and NPVs were artificially high due to the large number of non-allergic patients included in the study population, we did not calculate these accuracy measures and no recommendations can be made for these accuracy measures.

In conclusion, this study showed that children diagnosed with asthma can be identified reliably with a range of medication proxies. The use of prescription data for the identification of children diagnosed with atopic dermatitis and allergic rhinitis is questionable, since sufficient PPVs were only yielded in combination with low sensitivity values. Data collection for childhood patients is challenging, and prescription databases may provide convenient and easily available sources. The broad spectrum of medication proxies presented in this study may aid various epidemiological studies with the identification of children diagnosed with allergic disorders in the future.

Open Access This article is distributed under the terms of the Creative Commons Attribution 4.0 International License (http:// creativecommons.org/licenses/by/4.0/), which permits unrestricted use, distribution, and reproduction in any medium, provided you give appropriate credit to the original author(s) and the source, provide a link to the Creative Commons license, and indicate if changes were made.

\section{References}

1. Visser ST, Schuiling-Veninga CC, Bos JH, de Jong-van den Berg LT, Postma MJ (2013) The population-based prescription database IADB.nl: its development, usefulness in outcomes research and challenges. Expert Rev Pharmacoecon Outcomes Res 13(3):285292

2. Zuidgeest MG, van Dijk L, Smit HA, et al. (2008) Prescription of respiratory medication without an asthma diagnosis in children: a population based study. BMC Health Serv Res 8:16

3. Bianchi M, Clavenna A, Sequi M, Bonati M (2011) Asthma diagnosis vs. analysis of anti-asthmatic prescriptions to identify asthma in children. Eur J Clin Pharmacol 67:967-968

4. Osborne ML, Vollmer WM, Johnson RE, Buist AS (1995) Use of an automated prescription database to identify individuals with asthma. J Clin Epidemiol 48:1393-1397

5. Pont LG, van der Werf GT, Denig P, Haaijer-Ruskamp FM (2002) Identifying general practice patients diagnosed with asthma and their exacerbation episodes from prescribing data. Eur J Clin Pharmacol 57(11):819-825

6. Furu K, Skurtveit S, Langhammer A, Nafstad P (2007) Use of anti-asthmatic medications as a proxy for prevalence of asthma in children and adolescents in Norway: a nationwide prescription database analysis. Eur J Clin Pharmacol 63(7): 693-698

7. Moth G, Vedsted P, Schiøtz P (2007) Identification of asthmatic children using prescription data and diagnosis. Eur J Clin Pharmacol 63(6):605-611

8. Örtqvist AK, Lundholm C, Wettermark B, Ludvigsson JF, Ye W, Almqvist C (2013) Validation of asthma and eczema in populationbased Swedish drug and patient registers. Pharmacoepidemiol Drug Saf 22(8):850-860

9. Bacharier LB, Guilbert TW (2012) Diagnosis and management of early asthma in preschool-aged children. J Allergy Clin Immunol 130(2):287-296

10. Chubak J, Pocobelli G, Weiss NS (2012) Tradeoffs between accuracy measures for electronic health care data algorithm. J Clin Epidemiol 65(3):343-349

11. Bantz SK, Zhu Z, Zheng T (2014) The atopic March: progression from atopic dermatitis to allergic rhinitis and asthma. J Clin Cell Immunol 5(2)

12. World Organisation of Family Doctors (WONCA) (1998) ICPC-2: International Classification of Primary Care, 2nd edn. Oxford University Press, Oxford

13. Schokker S, Groenhof F, van der Veen WJ, van der Molen T (2010) Prescribing of asthma medication in primary care for children aged under 10. Prim Care Respir J 19(1):28-34

14. Schrijvers AJP (ed) (1997) Health and health care in the Netherlands: a critical self-assessment of Dutch experts in medical and health sciences. De Tijdstroom, Utrecht, The Netherlands

15. Van-der-Werf GT, Smith RJA, Stewart RE, Meyboom-de Jong B (1998) Spiegel op de huisarts: over registratie van ziekte, medicatie en verwijzingen in de geautomatiseerde huisartspraktijk. Groningen, The Netherlands

16. Pont LG, Denig P, van der Molen T, van der Veen WJ, HaaijerRuskamp FM (2004) Validity of performance indicators for assessing prescribing quality: the case of asthma. Eur J Clin Pharmacol 59(11):833-840 Epub 2003 Nov 18

17. NHG (Dutch General Practitioner Guidelines). https://www.nhg. org/standaarden/volledig/nhg-standaard-astma-bij-kinderen. Last accessed 24th April 2015.

18. NHG (Dutch General Practitioner Guidelines). https://www.nhg. org/standaarden/volledig/nhg-standaard-constitutioneel-eczeem. Last accessed 24th April 2015.

19. NHG (Dutch General Practitioner Guidelines). https://www.nhg. org/standaarden/volledig/nhg-standaard-allergische-en-nietallergische-rhinitis. Last accessed 24th April 2015.

20. Asher MI, Montefort S, Björkstén B, Lai CK, Strachan DP, Weiland SK, Williams H (2006) ISAAC phase three study 
group. Worldwide time trends in the prevalence of symptoms of asthma, allergic rhinoconjunctivitis, and eczema in childhood: ISAAC phases one and three repeat multicountry cross-sectional surveys. Lancet 368(9537):733-743
21. Mulder B, Schuiling-Veninga CC, Bos HJ, De Vries TW, Jick SS, Hak E (2014) Prenatal exposure to acid-suppressive drugs and the risk of allergic diseases in the offspring: a cohort study. Clin Exp Allergy 44(2):261-269 